\title{
10. Cardiología crítica
}

\subsection{Ruptura de septum interventricular en síndrome coronario agudo \\ Rodríguez López Sergio Alberto,}

Rivera Lara Pedro, Antezana Castro Javier, Almeida Gutiérrez Eduardo, Gutiérrez Cuevas Luis Manuel

Hospital de Cardiología del Centro Médico Nacional Siglo

XXI del Instituto Mexicano del Seguro Social.

Tipo de estudio: Reporte de casos clínicos con revisión de fuentes

Introducción: La ruptura del septum ventricular (RSV) ocurre en el $0.2 \%$ de los infartos agudos de miocardio (IAM), 46\% de los casos causados por afección de la arteria coronaria derecha, cuya ruptura usualmente es más compleja envolviendo la pared libre. Descripción del caso: Mujer de 72 años, tabaquismo intenso y sedentarismo. Acude a nuestro hospital con dolor precordial típico, electrocardiograma (ECG) con hallazgo de desnivel positivo del ST en derivaciones inferiores. Presenta hipotensión y soplo en barra, se realiza ecocardiografía transtorácica que muestra ruptura del septum ventricular porción inferoseptal a nivel del segmento apical. Metodología: Se realizó una búsqueda en PubMed utilizando la palabra clave interventricular septal rupture, se limitó la búsqueda a los últimos 10 años, se incluyeron artículos de revisión, ensayos clínicos y metaanálisis. Resultados: Se realiza coronariografía con hallazgo de enfermedad coronaria significativa de múltiples vasos, se coloca balón intraaórtico de contrapulsación y vasopresor por choque cardiogénico e ingresa a cirugía de urgencia. Se coloca parche de teflón para cierre del defecto, plastia de Alfieri mitral y revascularización egresando con soporte mecánico y vasopresor farmacológico. Discusión: La RSV genera shunt izquierda a derecha, sobrecarga de volumen y presión

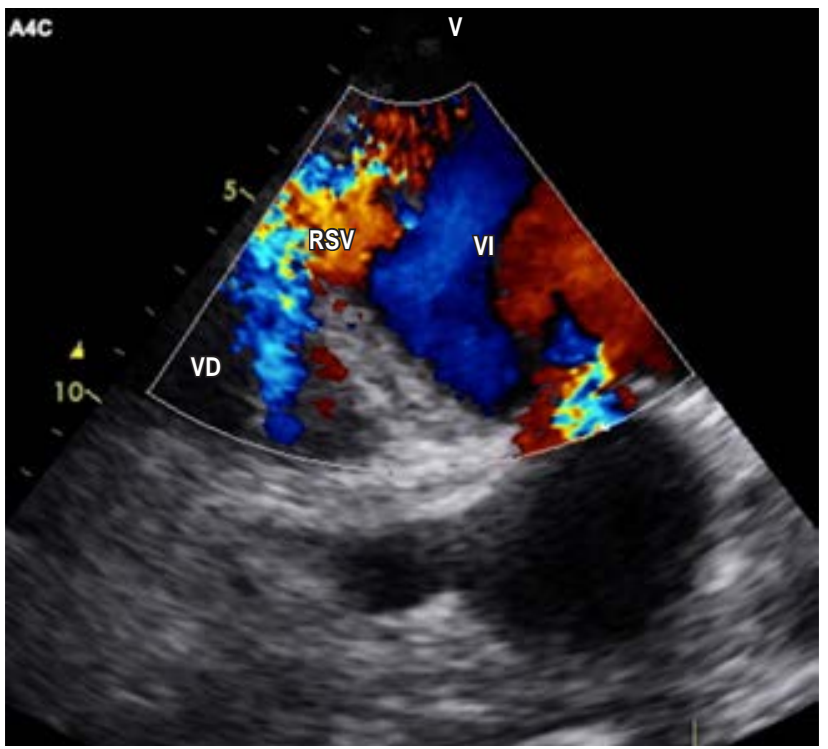

Figura 10.1.1.

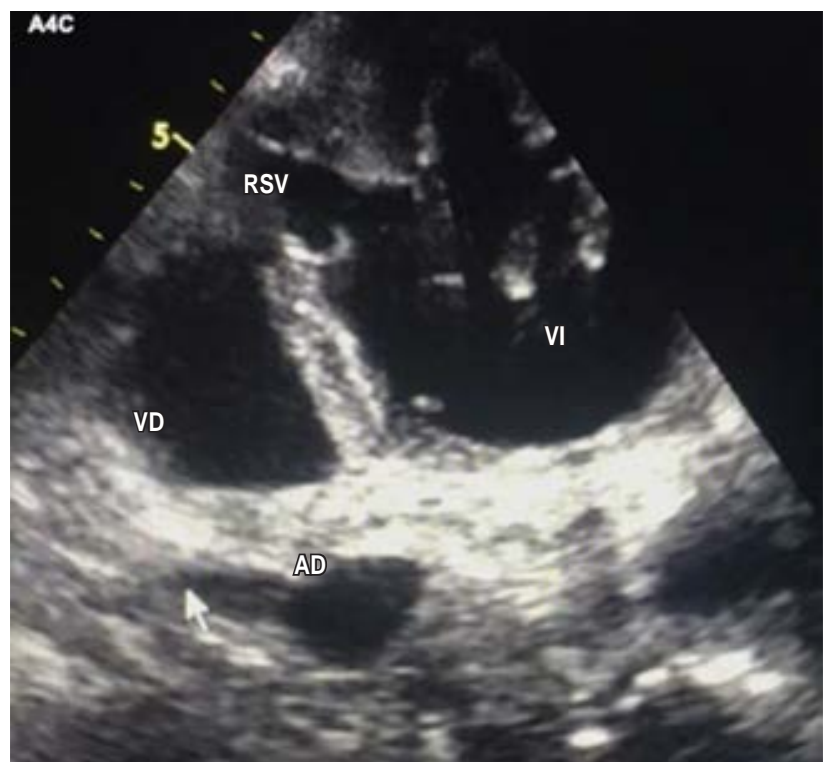

Figura 10.1.2.

del ventrículo derecho, aumento del retorno venoso pulmonar y sobrecarga izquierda secundaria. La mortalidad quirúrgica es del 60\% en las primeras 24 horas. Se reduce a 10\% después de 21 días y aumenta al 94\% sin intervención después de 30 días.

\subsection{Panvasculitis linfocítica activa como causa no aterosclerosa de síndrome isquémico coronario agudo}

Esparza Pérez Denhi,*

Robles Rangel Marco Antonio, ${ }^{\ddagger}$ Almeida Gutiérrez Eduardo, ${ }^{\ddagger}$

Lupercio Mora Karina, ${ }^{\ddagger}$ Pacheco López Alejandro, ${ }^{\ddagger}$ Solís Cancino Marlén, ${ }^{\ddagger}$

Arellano Escudero Octavio ${ }^{\ddagger}$

* Hospital de Cardiología del Centro Médico Nacional Siglo

XXI, IMSS. ${ }^{\ddagger}$ Instituto Mexicano del Seguro Social.

Tipo de estudio: Reporte de casos clínicos con revisión de fuentes

Introducción: La arteritis coronaria, en el contexto de panvasculitis activa, es una afección reumática con presentación atípica poco reconocida. El desafío es aún mayor al tratarse de un paciente joven. El espectro de las enfermedades coronarias no ateroscleróticas representa el $5 \%$ del infarto agudo de miocardio mortal, y en escenarios clínicos críticos deben tomarse decisiones rápidas y acertadas. Descripción del caso: Hombre de 31 años que consulta por dolor precordial y diaforesis. El electrocardiograma actual es poco concluyente, troponina alta. Presentó fibrilación ventricular durante la angiografía coronaria, tras 40 minutos de reanimación cardiopulmonar fallece. Análisis histopatológico: actividad linfocitaria, inflamación transmural 


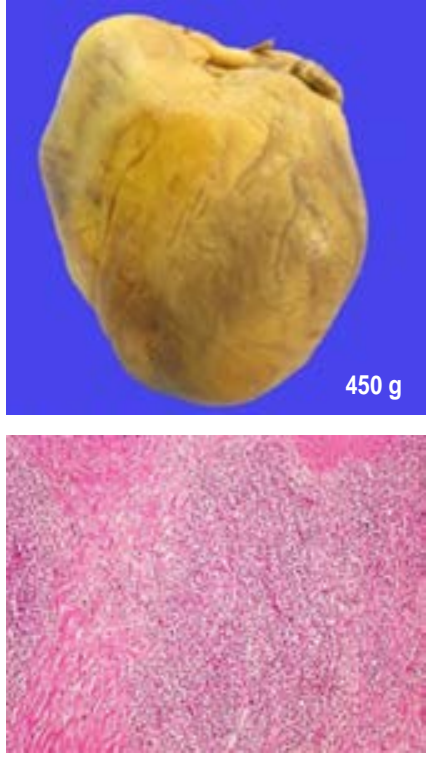

Infiltrado linfocitario, capa íntima

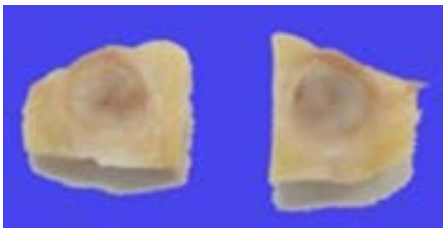

Tronco coronario izquierdo

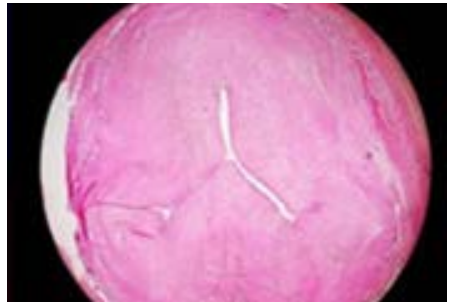

Tronco coronario izquierdo
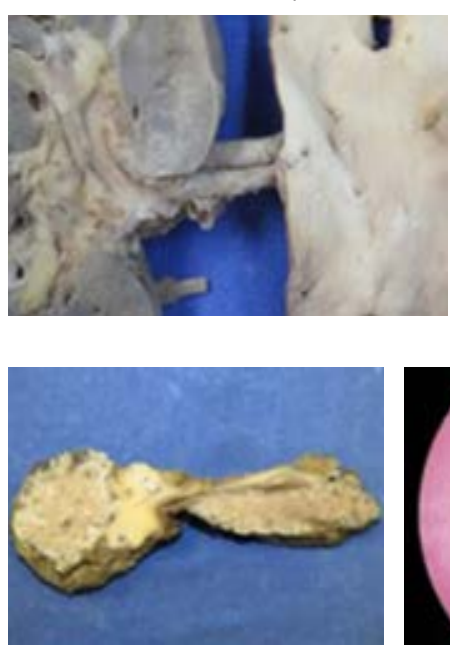

Páncreas
Figura 10.2.1.

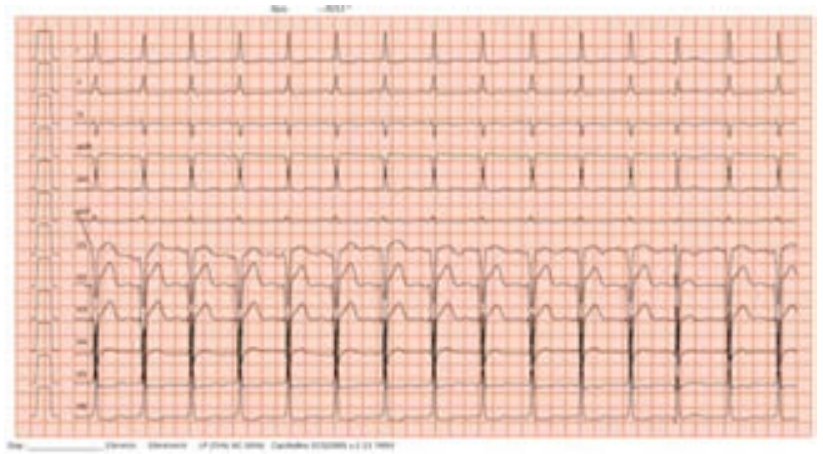

Figura 10.2.2.

en la íntima y necrosis fibrinoide, condicionando oclusión mayor al $90 \%$ de la luz de las arterias epicárdicas, infiltrado linfocitario en otras arterias. Metodología: Se realizó búsqueda de términos MeSH panvasculitis, vasculitis linfocitaria, ischemia in the young en las bases de datos con repositorios de evidencia científica arbitrada indexa-

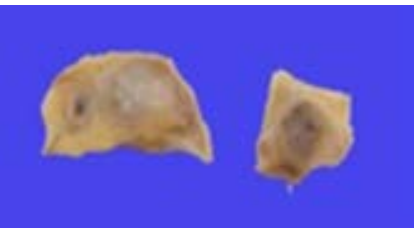

Circunfleja

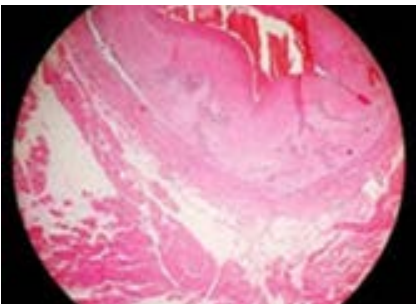

Circunfleja

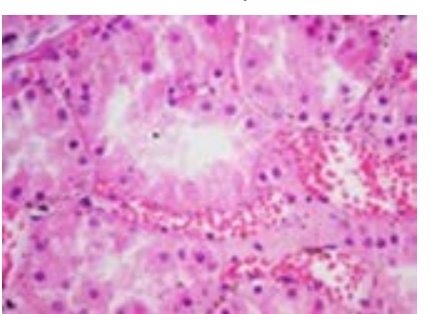

Arteria renal

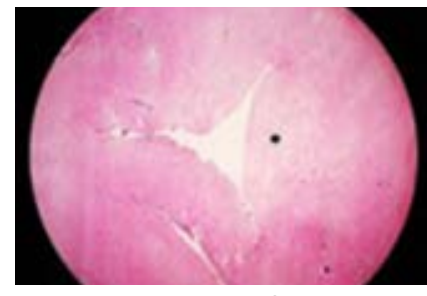

Arteria pancreática

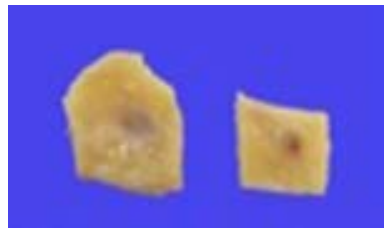

Coronaria derecha

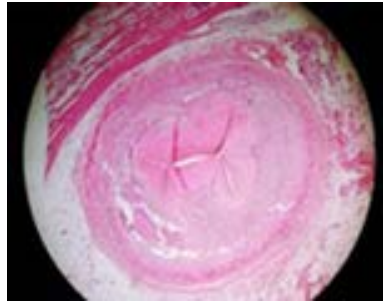

Coronaria derecha
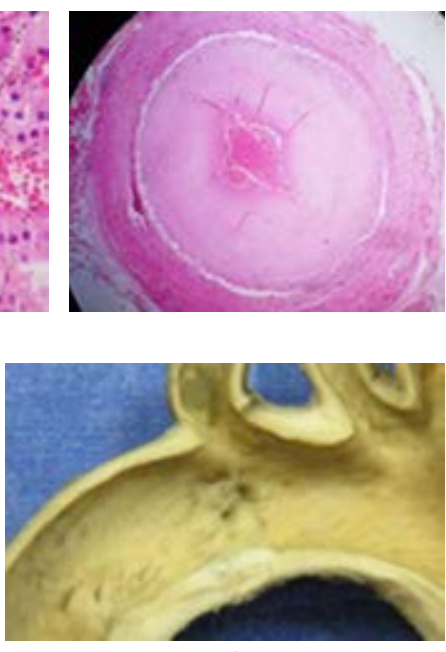

Arco aórtico das (PubMed, EBSCO, Ovid, ClinicalKey) y no indexadas (Google Scholar). Resultados: El espectro de las enfermedades coronarias no ateroescleróticas representa el 5\% del infarto agudo de miocardio mortal. La arteritis coronaria es una entidad de comportamiento heterogéneo como expresión de tormenta inflamatoria en el contexto de actividad reumatológica y es una complicación potencialmente mortal, pero si el diagnóstico se realiza oportunamente, permite tratar la enfermedad correctamente, cambiando por completo el pronóstico. Discusión: Este caso nos deja como enseñanza que pese a que la enfermedad ateroesclerosa es la principal causa ante un cuadro clínico de angina, podrán existir otras etiologías, más aún al tratarse de pacientes jóvenes. Por ello, es importante considerar otros fenómenos fisiopatológicos para vislumbrar la etiología de manera certera.

\subsection{Soporte mecánico circulatorio con ECMO VA en infarto del ventrículo derecho}

Patricio Balbuena Roney Abinain,

Gómez Gómez Carlos, Ochoa Salinas Jacqueline, Lupercio Mora Karina,

Almeida Gutiérrez Eduardo

Hospital de Cardiología del Centro Médico Nacional Siglo XXI.

Tipo de estudio: Reporte de casos clínicos con revisión de fuentes 
Figura 10.3.1.
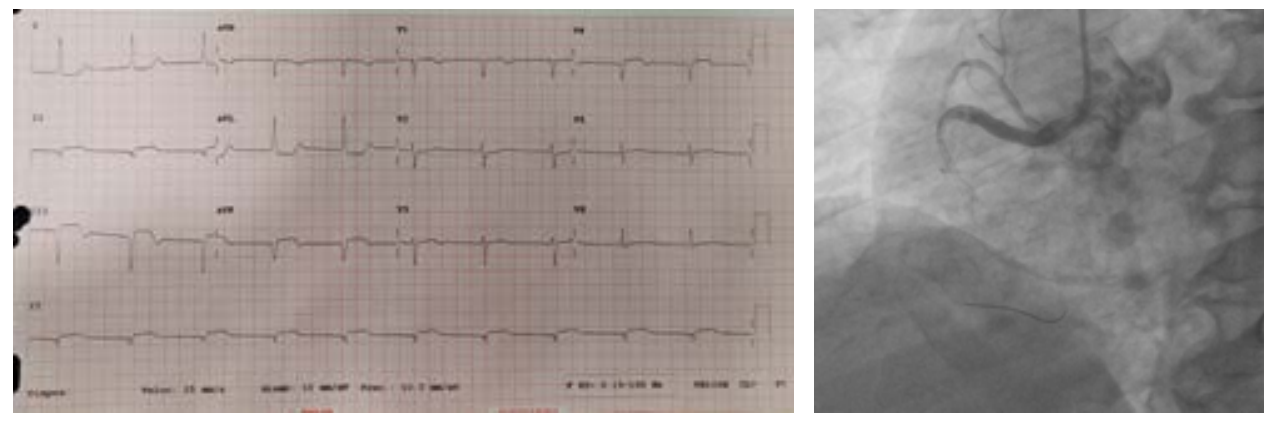

Introducción: El choque cardiogénico (CC) sigue siendo una de las principales causas de muerte para el infarto agudo de miocardio. El manejo convencional conlleva tasas de mortalidad superiores al 50\%. Se han desarrollado y estudiado dispositivos de asistencia ventricular incluyendo ECMO (membrana de circulación extracorpórea) en el contexto CC en síndrome isquémico agudo. Descripción del caso: Mujer de 69 años, hipertensa y dislipidemia. Presenta dolor torácico e hipotensión. Electrocardiograma: desnivel positivo del ST inferior con extensión eléctrica al ventrículo derecho. Coronariografía: coronaria derecha, segmento proximal con oclusión total aguda, único vaso enfermo; intervencionismo coronario percutáneo fallido. Presentó CC refractario colocando ECMO VA con respuesta favorable a las 2448 horas, retirado a los ocho días. Metodología: A través de método booleano se realizó búsqueda de términos MeSH venoarterial extracorporeal membrane oxygenation y Right Ventricular Myocardial Infarction en las bases de datos con repositorios de evidencia científica arbitrada indizada (PubMed) y no indizadas (Google Scholar). Resultados: Existe poca evidencia ECMO VA de canulación periférica, como soporte ventricular derecho, el tratamiento estándar es actualmente la canulación de la vena femoral o aurícula derecha y la arteria pulmonar. ECMO VA mediante canulación periférica es un enfoque alternativo, menos invasivo, sin requerir esternotomía, que permite la asistencia ventricular en choque cardiogénico. Discusión: Este caso muestra la presentación choque cardiogénico por un infarto al ventrículo derecho con repercusión hemodinámica que requirió apoyo circulatorio con ECMO VA. No existe evidencia solida del uso de ECMO VA con canulación periférica para infarto al ventrículo derecho con repercusión hemodinámica, pero puede ser una alternativa que disminuya la mortalidad.

\subsection{Perfil protrombótico en pacientes con COVID-19 atendidos en el IMSS}

Borrayo Sánchez Gabriela,* Hernández González Martha Alicia, ${ }^{\ddagger}$

Flores Morales Abelardo, ${ }^{\S}$ Romero Gutiérrez Laura, ${ }^{\S}$ Anda Garay Carlos ${ }^{\S}$

* Instituto Mexicano del Seguro Social. ${ }^{*}$ UMAE HE, IMSS,

León, Guanajuato. ${ }^{\S}$ UMAE Hospital de Especialidades

del Centro Médico Nacional Siglo XXI, IMSS.

Tipo de estudio: Estudio Pronóstico

Introducción: La pandemia por COVID-19 ha afectado la salud en todo el mundo, México ocupa el tercer lugar en muertes (11\%) ante ausencia de vacunas y tratamientos efectivos; se acepta el uso compasivo terapéutico con trazadores de algoritmos interinos, la mayor severidad se relaciona con un estado de hiperinflamación y trombosis. Objetivo: Evaluar el perfil protrombótico en pacientes con COVID-19 que demandan atención de urgencias, hospitalización o terapia intensiva atendidos con trazadores de algoritmos interinos en el IMSS. Metodología: Diseño
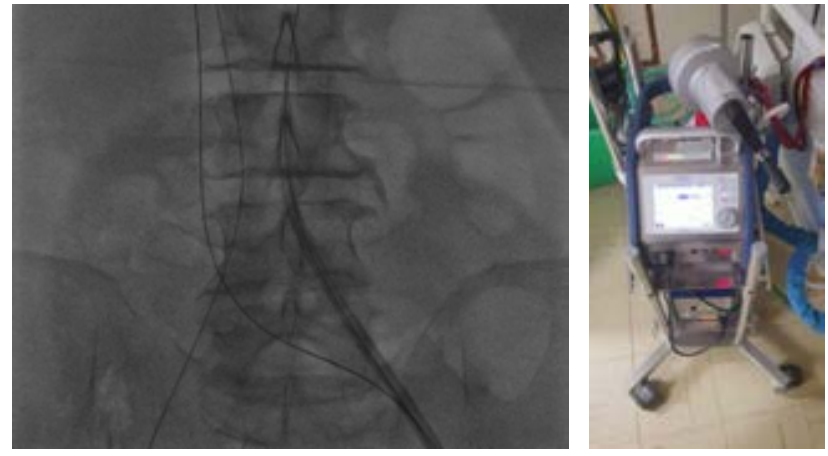

Figura 10.3.2.

ambispectivo observacional, para evaluar el perfil protrombótico y el efecto del uso compasivo de algoritmos interinos a través de trazadores diseñados por expertos en comparación con el manejo estándar para pacientes con COVID-19 en unidades médicas del IMSS. Resultados: Incluimos 312 casos, 58\% hombres y $42 \%$ mujeres, edad promedio $55 \pm 14$ años, IMC $29.3 \pm 4.9$, los factores de riesgo fueron: hipertensión $39.4 \%$, diabetes $30.8 \%$, obesidad $20.5 \%$, tabaquismo $11.5 \%$, dislipidemia 7.4\% e inmunosupresión 3.8\%. La severidad COVID-19: leve en $9.9 \%$, moderada $32.7 \%$ y severa $35.3 \%$. El perfil inflamatorio y protrombótico se manifestó: PCRhs $15.2 \pm 12.1 \mathrm{mg} / \mathrm{dL}$, deshidrogenasa láctica $398 \pm 186 \mathrm{U} / \mathrm{L}$, fibrinógeno $820 \pm 267 \mathrm{mg} / \mathrm{dL}$, ferritina 1,095 \pm 960 ng/mL, dímero D, 1200 g/L (q25 449, q75 3353). La mortalidad fue del $16 \%$, se asoció significativamente a la gravedad y a un estado protrombótico $(r=0.248 p<0.0001)$. Discusión y conclusiones: La población es de alto riesgo, el 68\% fue COVID-19 moderado-severo, con un perfil inflamatorio y protrombótico, se asoció significativamente a mayor mortalidad y es un objetivo terapéutico actual.

\subsection{Efecto de redes institucionales en la reperfusión coronaria bajo el protocolo Código Infarto IMSS}

Borrayo Sánchez Gabriela,* Parra Michel Rodolfo, ${ }^{\ddagger}$

Ramírez Arias Erick, ${ }^{\S}$ Barraza Félix Rafael,`

Hernández González Martha Alicia,, Betancourt Hernández Lidia**

* Instituto Mexicano del Seguro Social. * UMAE Hospital de

Especialidades CMNO, IMSS. ${ }^{\S}$ UMAE Hospital de Cardiología del Centro Médico Nacional Siglo XXI, IMSS. ^ UMAE Hospital de Especialidades

La Raza, IMSS. || UMAE Hospital de Especialidades, IMSS, León

Guanajuato. ** UMAE Hospital de Especialidades, IMSS, Veracruz.

Tipo de estudio: Registros locales o nacionales 
Introducción: La reperfusión con redes de atención mejora el pronóstico y reduce la mortalidad en pacientes con infarto agudo de miocardio con elevación del ST (IAM CEST). México tiene la mayor mortalidad en los países de la OCDE. Objetivo: Evaluar la mejora en el tratamiento del IAM CEST en uno de los sistemas de salud más grandes de América Latina con el protocolo de atención Código Infarto. Metodología: Estudiamos pacientes consecutivos del RENASCA IMSS con IAM CEST de $<12$ horas de evolución, con un diseño antes y después (grupo I antes y grupo II después del protocolo Código Infarto). Se aplicó en 23 redes de atención con herramientas tecnológicas, incluyendo 344 unidades de los tres niveles de atención, con énfasis en el diagnóstico temprano y reperfusión oportuna. Resultados: Incluimos 35,945 pacientes consecutivos de 2014 a 2019; 27,445 hombres (76.9\%) y 8,500 mujeres (23.1\%), edad promedio $62.8 \pm 11.8$ años. Del grupo I: 20,081 casos y del grupo II: 15,864. Mejoró la reperfusión entre los grupos I y II: intervención coronaria percutánea (11 a 28.2\%), terapia fibrinolítica (21.5 a 33.9\%), la estrategia farmacoinvasiva (1 a 8.5\%) y la no reperfusión bajó (66.5 a 29.5\%), ( $p<0.0001)$. Disminuyeron los tiempos puerta aguja 138 a 83 minutos $p<$ 0.0001, puerta balón 189 a 94 minutos p < 0.0001, y la mortalidad temprana (19.4 contra 8.2\% OR: 0.37, p < 0.0001). Discusión y conclusiones: Las redes de Código Infarto IMSS mejoraron la reperfusión, disminuyeron los tiempos de atención y las complicaciones, incluyendo la muerte; hoy puede ser la estrategia universal en nuestro país. 